\title{
Optical matrix element in InAs/GaAs quantum dots: Dependence on quantum dot parameters
}

\author{
A. D. Andreev \\ Department of Physics, University of Surrey, Guildford, GU2 7XH, United Kingdom \\ E. P. O'Reilly \\ Tyndall National Institute, Lee Maltings, Cork, Ireland
}

(Received 13 May 2005; accepted 3 October 2005; published online 15 November 2005)

\begin{abstract}
We present a theoretical analysis of the optical matrix element between the electron and hole ground states in InAs/GaAs quantum dots (QDs) modeled with a truncated pyramidal shape. We use an eight-band $\mathbf{k} \cdot \mathbf{p}$ Hamiltonian to calculate the QD electronic structure, including strain and piezoelectric effects. The ground state optical matrix element is very sensitive to variations in both the QD size and shape. For all shapes, the matrix element initially increases with increasing dot height, as the electron and hole wave functions become more localized in $k$ space. Depending on the QD aspect ratio and on the degree of pyramidal truncation, the matrix element then reaches a maximum for some dot shapes at intermediate size beyond which it decreases abruptly in larger dots, where piezoelectric effects lead to a marked reduction in electron-hole overlap. (c) 2005 American Institute of Physics. [DOI: 10.1063/1.2130378]
\end{abstract}

Semiconductor quantum dots (QDs) have been widely studied because of their unique fundamental properties and also because they are considered to be promising candidates for a new generation of semiconductor laser. ${ }^{1}$ One of the main advantages initially proposed for QDs was the atomiclike (zero-dimensional) density of states, which should provide a relatively large optical gain at a reduced carrier density. ${ }^{2}$ In real QD structures the gain is, however, significantly reduced due both to inhomogeneous broadening of the atomic-like density of states and also because of a reduced optical matrix element. This can even prohibit lasing from the ground state in some QD structures. ${ }^{3-5}$ Previous theoretical studies ${ }^{6,7}$ suggested that the low gain arises because the built-in piezoelectric field leads to the ground state hole wave function being elongated, with the matrix element then reduced due to lower overlap between the electron and hole wave functions. We show here that this is not the only reason for low optical matrix element. The QDs in Refs. 6 and 7 were assumed to be pyramidal with a base to height aspect ratio of 2. In real structures the typical QD shape is markedly different, strongly influencing the electron and hole wave functions, ${ }^{8}$ and thereby modifying the optical matrix element. Previous theoretical studies of InAs/GaAs QDs either do not contain calculations of the optical matrix element ${ }^{8,9}$ or consider only one specific QD shape. ${ }^{10}$

The aim of this letter is to identify the factors determining the ground state optical matrix element in QDs, and its dependence on QD shape and size. In a bulk semiconductor the optical matrix element decreases for transitions away from the Brillouin zone center, and also depends on the wave vector direction with respect to the light polarization. This fundamental property of semiconductors, as well as the piezoelectric effect, causes the matrix element in QDs to be very sensitive to variations in the QD geometrical parameters, leading to a lower optical matrix element in QDs compared to the maximum "ideal" band edge value of a bulk semiconductor.

To calculate the electronic structure of the QDs we use a plane-wave expansion method, assuming a periodic array of widely separated QDs. ${ }^{11}$. The wave function of each electron or hole is described by a linear combination of bulk states

$$
\Psi(\mathbf{r})=\sum_{\mathbf{k}, \alpha} C_{\mathbf{k}, \alpha}|\mathbf{k}, \alpha\rangle,
$$

where $|\mathbf{k}, \alpha\rangle \propto \exp (i \mathbf{k r})$ is an eigenstate of the bulk 8 $\times 8 \mathbf{k} \cdot \mathbf{p}$ Hamiltonian given in Ref. 12, and $\alpha$ denotes electron, heavy-, light-, or spin-split-off holes (including spin). The electron and hole energies in the QDs and the coefficients $C_{\mathbf{k}, \alpha}$ are found by diagonalizing a large matrix, whose matrix elements depend on the Fourier transform of the QD shape, strain, and piezoelectric field distribution. ${ }^{8,11,13}$ For a given light polarization, the optical matrix element $M_{\text {eh }}$ between an electron and hole state in a QD is given by

$$
M_{\mathrm{eh}}=\sum_{\mathbf{k}, \alpha, \beta}\left[C_{\mathbf{k}, \alpha}^{(e)}\right]^{*} C_{\mathbf{k}, \beta}^{(h)} M_{\alpha \beta}(\mathbf{k}),
$$

where $M_{\alpha \beta}(\mathbf{k})$ is the optical matrix element between two bulk states $\alpha$ and $\beta$ at wave vector $\mathbf{k}$, and $C_{\mathbf{k}, \alpha}^{(e)}$ and $C_{\mathbf{k}, \beta}^{(h)}$ are the coefficients defined by Eq. (1) describing the electron and hole QD states. Since the coefficients in Eq. (1) are normalized, $\Sigma_{\mathbf{k}, \alpha}\left|C_{\mathbf{k}, \alpha}^{(e, h)}\right|^{2}=1$, we immediately conclude from Eq. (2) that the squared optical matrix element in a QD is always smaller than that at $\mathbf{k}=0$ in a bulk or quantum well material.

We consider InAs QDs with a truncated pyramidal shape. ${ }^{8}$ The QD size and shape can be described by three independent parameters; namely the QD height $h$, the aspect ratio $\alpha=b_{b} / h$, and the truncation degree $\eta=b_{t} / b_{b}$ (the QD bottom and top are $b_{b} \times b_{b}$ and $b_{t} \times b_{t}$ squares, respectively); for a full pyramid we have $\eta=0$, while $\eta=1$ for a QD box. For the QDs under consideration the ground state matrix element is much larger for light polarized in the QD $x-y$ plane than it is for light polarized along the growth direction $z$. We therefore set the light polarization vector along the $x$ axis. Keeping in mind the main application of the present study for gain calculations, we consider the quantity $\left|M_{\text {tot }}\right|^{2}$, which is the modulus squared of the optical matrix element 


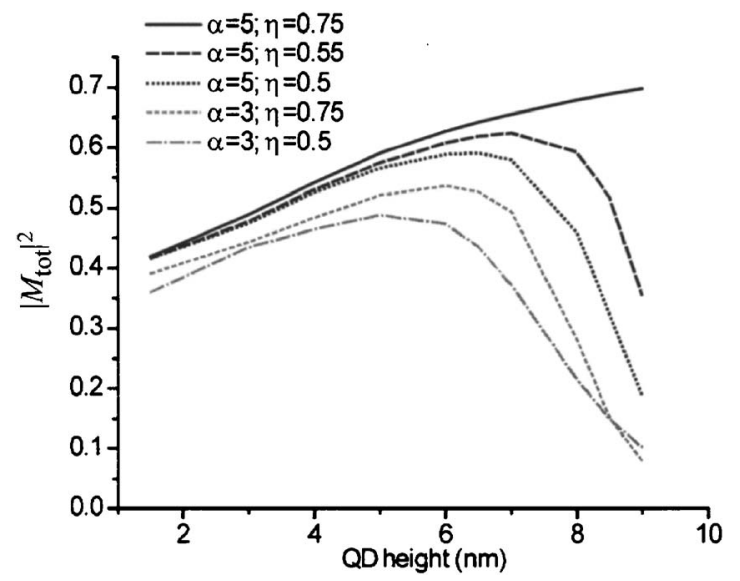

FIG. 1. Variation with dot height, $h$, of the modulus squared of the optical matrix element $\left|M_{\text {tot }}\right|^{2}$ for the ground state electron-hole transition in InAs/GaAs QDs of truncated pyramidal shape, summed over degenerate spin states. The value of $\left|M_{\text {tot }}\right|^{2}$ is given in units of $P_{0}^{2}$ [where $P_{0}$ is the interband momentum matrix element, $P_{0}=\left(\hbar / m_{0}\right)\left\langle s\left|p_{x}\right| x\right\rangle$, see Ref. 11]. The light polarization vector is taken along the $x$ direction.

for the electron-hole ground state transition summed over degenerate spin states. Figure 1 shows the calculated variation of $\left|M_{\text {tot }}\right|^{2}$ with dot height for five different shapes. $\left|M_{\text {tot }}\right|^{2}$ initially increases with dot height, in four of the five cases reaching a peak value beyond which it decreases rapidly. We also note that in each case the value of $\left|M_{\text {tot }}\right|^{2}$ is noticeably smaller than the maximum value of unity which might be expected for an "ideal" QD. The main aim of this letter is to identify the causes of this behavior.

It can be seen from Eq. (2) that the magnitude of the optical matrix element is determined by two factors: (i) the dependence of the bulk matrix element $M_{\alpha \beta}$ on $\mathbf{k}$, and (ii) how the coefficients $C_{\mathbf{k}, \alpha}^{(e)}$ and $C_{\mathbf{k}, \alpha}^{(h)}$ overlap in $\mathbf{k}$ space with each other and with $M_{\alpha \beta}(\mathbf{k})$. This overlap is very sensitive to the QD size and shape. The hole wave function consists of mainly two components: a heavy-hole $(\mathrm{HH})$ contribution and a light-hole (LH) contribution [each is represented by a summation in Eq. (1) over the corresponding set of bulk states]. For the hole ground state, the admixture of LH bulk states is typically about $10 \%-20 \%$, depending on the QD geometry. We therefore concentrate our analysis on the electron-HH component. The dependence of the bulk matrix element $\left|M_{\alpha \beta}\right|^{2}$ on $\mathbf{k}$ for the electron-heavy-hole transition is illustrated in Figs. 2(a) and 2(b) The optical matrix element is calculated following the method developed in Ref. 14. For the electron-heavy-hole bulk states, the maximum matrix element is achieved for a given $|\mathbf{k}|$ when $\mathbf{k}$ is perpendicular to the light polarization vector $\mathbf{e}=\mathbf{e}_{x}$ [see Fig. 2(b)]. In addition, the bulk matrix element $\left|M_{\mathrm{E}-\mathrm{HH}}\right|^{2}$ decreases quite rapidly as $|\mathbf{k}|$ increases [Fig. 2(a)]. For example when $k_{\mathrm{y}}=0.46 \mathrm{~nm}^{-1}$ (which corresponds to a distance in real space of $2 \pi / k$ $\sim 13.7 \mathrm{~nm}$ ), the InAs bulk matrix element summed over spin states is only 0.5 of its maximum value at $k=0$. This reduction is due predominantly to band-mixing effects, and so is best described in a $\mathbf{k} \cdot \mathbf{p}$ model that includes both the conduction and valence bands, along with corrections due to other bands. ${ }^{14}$ The effect of decreasing matrix element with the in-plane momentum $k_{\|}$is well-known in quantum well structures. ${ }^{15}$ For example, the calculated optical matrix element for E-HH transitions in an InGaAs quantum well decreased by more than a factor of three when $k_{\|}$increased Downloaded 30 Mar 2009 to 131.227 .178 .132 . Redistribution subject

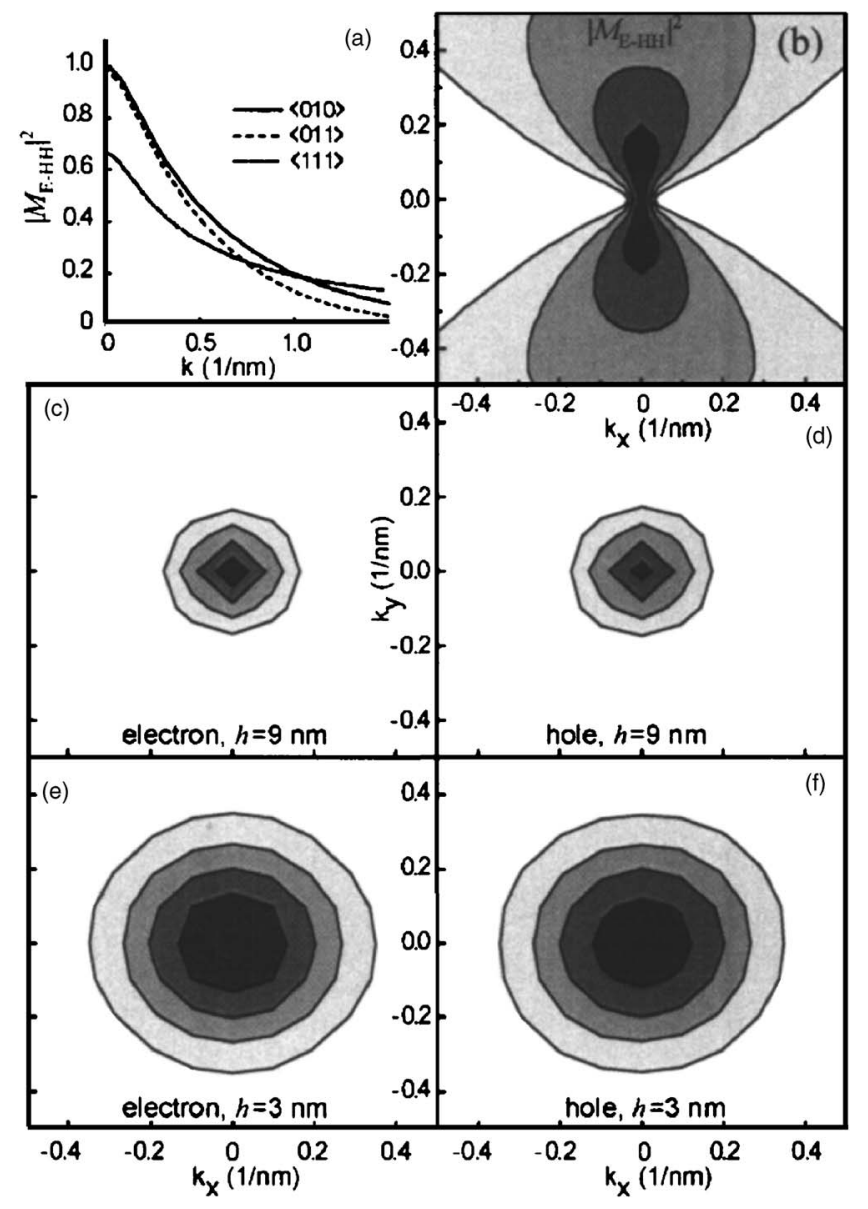

FIG. 2. (a) Modulus squared of the optical matrix element for electronheavy hole transitions $\left|M_{\mathrm{E}-\mathrm{HH}}\right|^{2}$ in bulk unstrained InAs (summed over degenerate spin states); units are the same as in Fig. 1, the light polarization vector is along the $x$ direction. (b) Contour plot of $\left|M_{\mathrm{E}-\mathrm{HH}}\right|^{2}$ in the $k_{x}-k_{y}$ plane for bulk InAs; (c)-(f) Contour plot of the wave function coefficients $\left|C_{\mathbf{k}, \alpha}^{(e)}\right|^{2}$ and $\left|C_{\mathbf{k}, \alpha}^{(h)}\right|^{2}$ in the $k_{x}-k_{y}$ plane for QDs with $\alpha=5, \eta=0.75$, and $h=3 \mathrm{~nm}$ (c), (d) and $h=9 \mathrm{~nm}(\mathrm{e}),(\mathrm{f})$. Five grades of grey color correspond to the values of $0 \%-20 \% ; 20 \%-40 \% ; 40 \%-60 \% ; 60 \%-80 \%$ and $80 \%-100 \%$ of the maximum value, respectively.

from 0 to $1 \mathrm{~nm}^{-1} \cdot{ }^{16}$ The significant decrease of $\left|M_{\mathrm{E}-\mathrm{HH}}\right|$ in Fig. 2(a) at relatively large wave vectors occurs when the kinetic energy of the electron approaches the band gap energy. This effect is therefore considerably more pronounced here than would be the case for bulk materials with larger band gap. The described properties of the E-HH bulk matrix element have important consequences for the matrix element in QDs. As the QD height decreases, the characteristic size of the electron and hole wave function in $k$ space becomes larger [compare Figs. 2(c) and 2(d) with Figs. 2(e) and 2(f)] and the overlap in $\mathbf{k}$ space between $C_{\mathbf{k}, \alpha}^{(e)}, C_{\mathbf{k}, \beta}^{(h)}$, and $M_{\alpha \beta}(\mathbf{k})$ becomes smaller: this can be clearly seen by comparing Fig. 2(b) with Figs. 2(c)-2(f). The terms with larger $|\mathbf{k}|$ [and consequently a smaller average $M_{\alpha \beta}$ in Eq. (2)] play a more important role in small QDs, leading to a reduction in the QD optical matrix element as the QD height decreases. Thus, the trend of increasing $\left|M_{\text {tot }}\right|^{2}$ with $h$ is a direct consequence of the $\mathbf{k}$ dependence of $\left|M_{\mathrm{E}-\mathrm{HH}}\right|^{2}$ in a bulk semiconductor. We discuss later how this trend is interrupted for some QD shapes by piezoelectric effects, which lead to a sharp decrease in $\left|M_{\text {tot }}\right|^{2}$ beyond a critical value of $h$, see Fig. 1 .

It can be seen in Fig. 2(b) that the unstrained bulk matrix element $\left|M_{\mathrm{E}-\mathrm{HH}}\right|^{2}$ has a strong angular dependence. This to AIP license or copyright; see http://apl.aip.org/apl/copyright.jsp 


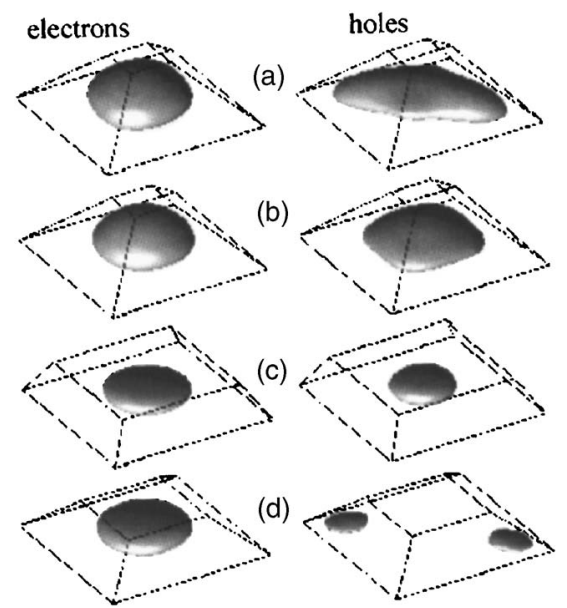

FIG. 3. Surfaces of constant probability density (equal to $35 \%$ of the maximum value) for the QD ground electron states (left-hand side) and ground hole states (right-hand side) for QDs of the following sizes: (a) $h=5.5 \mathrm{~nm}$, $\alpha=2.9, \eta=0.25$; (b) the same shape and size as (a), but without piezoelectric field; (c) $h=9 \mathrm{~nm} ; \alpha=5, \eta=0.75$; and (d) $h=9 \mathrm{~nm} ; \alpha=5, \eta=0.5$.

arises due to the change in the relative contribution of the valence $|x\rangle,|y\rangle$, and $|z\rangle$ states to the unstrained bulk $\mathrm{HH}$ band along different directions. Interband optical transitions with $\mathbf{e}_{x}$ polarization require valence states with $|x\rangle$ character. In the unstrained bulk material, the $\mathrm{HH}$ state has no $|x\rangle$ character along the $k_{x}$ direction: therefore giving zero matrix element, see Fig. 2(b). The anisotropy is reduced in quantum well and in biaxially strained structures. The reduction in symmetry splits the degeneracy of the HH and LH states at the valence band maximum, thereby reducing the ability to mix $|z\rangle$-like character from the LH states into the uppermost $\mathrm{HH}$ band. The relative contribution to the highest hole state of the $|z\rangle$ Bloch components can then provide a quantitative measure of the anisotropy effect displayed in Fig. 2(b). ${ }^{17}$ When the unstrained bulk HH band is averaged over all directions, the valence states have equal contributions of about $33 \%$ from each of $|x\rangle,|y\rangle$, and $|z\rangle$. The anisotropy observed in Fig. 2(b) is eliminated for a bulk material which is biaxially strained in the $z$ direction, with the valence $|z\rangle$ component then making nearly zero contribution to the uppermost valence states. For the various QDs we considered the situation is between these two extreme cases and the $|z\rangle$ terms account for $7 \%-20 \%$ of the ground state hole wave function. This is a significant contribution to the overall reduction in matrix element, although not as large as might be expected based on the bulk matrix elements presented in Fig. 2(b).

The piezoelectric field has previously been considered as the main cause of a reduced matrix element in pyramidal QDs. ${ }^{6}$ Due to this field, the hole wave function becomes elongated and asymmetric in the $x-y$ plane, leading to a decrease of the electron-hole overlap in real space. This situation is illustrated in Fig. 3(a). To demonstrate that the piezoelectric field need not be the prime cause of a reduced matrix element in medium-sized QDs (with $h \sim 5.5-6.5 \mathrm{~nm}$ ), we set the piezoelectric constants to zero in a test calculation on the QD of Fig. 3(a). The hole wave function is indeed more symmetric without the piezoelectric field [Fig. 3(b)], which visually increases the overlap between the electron and hole wave functions. However, the calculated total matrix element
$\left|M_{\text {tot }}\right|^{2}$ increases only from 0.447 to 0.471 (in units of $P_{0}^{2}$, see caption to Fig. 1). The situation changes, however, when the dot size is increased. Depending on the dot shape, the hole wave function either remains nearly symmetric [Fig. 3(c)] or becomes strongly localized in two potential pockets [Fig. 3(d)]. This localization leads to a steep drop in the matrix element $\left|M_{\text {tot }}\right|^{2}$ beyond a critical dot size for some QD shapes (see Fig. 1). The piezoelectric field creates two potential pockets for holes, with the size and depth of these pockets determined by the QD geometry. In InAs QDs we find that the piezoelectric potential pockets lose their ability to bind a hole for flat QDs $(\alpha=5, \eta \geqslant 0.75)$, and the hole wave function remains almost symmetric even for quite large QDs $(h$ $=10 \mathrm{~nm}$ ). The effect of the piezoelectric field on the optical matrix element in large QDs then depends on the QD shape. For many shapes this leads to a steep decrease of $\left|M_{\text {tot }}\right|^{2}$ beyond a critical QD height (Fig. 1); however, for other shapes (with a large aspect ratio $\alpha$ and truncation factor $\eta$ ), the piezoelectric field has little effect on the QD ground state matrix element.

In summary, we have analyzed theoretically the ground state optical matrix element in QDs. For many dots the k dependence of the bulk optical matrix element contributes to a reduction in matrix element compared to that initially expected in an ideal QD. The magnitude of the matrix element is very sensitive to variations in QD size and shape. It should therefore be possible to engineer the size and shape of QDs with the aim to optimize the optical gain in QD lasers and optical amplifiers. In particular, "flat" QDs with a large aspect ratio have a larger matrix element. We conclude that, depending on the QD geometry, the piezoelectric field may either have little effect on the ground state matrix element or else will markedly reduce it, effectively switching off the ground state optical transition in some QDs.

This work was supported by SFI (Ireland).

${ }^{1}$ D. Bimberg, M. Grundmann, and N. N. Ledentsov, Quantum Dot Heterostructures (Wiley, New York, 1999).

${ }^{2}$ M. Asada, Y. Miyamoto, and Y. Suematsu, IEEE J. Quantum Electron. QE-22, 1915 (1986).

${ }^{3}$ M. V. Maximov et al., Electron. Lett. 35, 2038 (1999).

${ }^{4}$ L. Harris, A. D. Ashmore, D. J. Mowbray, M. S. Skolnick, M. Hopkinson, G. Hill, and J. Clark, Appl. Phys. Lett. 75, 3512 (1999).

${ }^{5}$ G. Park, O. B. Shchekin, S. Csutak, D. L. Huffaker, and D. G. Deppe, Appl. Phys. Lett. 75, 3267 (1999).

${ }^{6}$ L. V. Asryan, M. Grundmann, N. N. Ledentsov, O. Stier, R. A. Suris, and D. Bimberg, J. Appl. Phys. 90, 1666 (2001).

${ }^{7}$ O. Stier, M. Grundmann, and D. Bimberg, Phys. Rev. B 59, 5688 (1999).

${ }^{8}$ J. A. Barker and E. P. O'Reilly, Phys. Rev. B 61, 13840 (2000).

${ }^{9}$ C. Pryor, Phys. Rev. B 60, 2869 (1999); J. Kim, L.-W. Wang, and A. Zunger, ibid. 57, R9408 (1998).

${ }^{10}$ W. Sheng and J.-P. Leburton, Appl. Phys. Lett. 80, 2755 (2002); W. Yang, H. Lee, T. J. Johnson, P. C. Sercel, and A. G. Norman, Phys. Rev. B 61, 2784 (2000).

${ }^{11}$ A. D. Andreev, Proc. SPIE 3284, 151 (1998); A. D. Andreev and E. P. O'Reilly, Phys. Rev. B 62, 15851 (2000).

${ }^{12}$ T. B. Bahder, Phys. Rev. B 41, 11992 (1990); 46, 9913 (1992).

${ }^{13}$ A. D. Andreev, J. R. Downes, D. A. Faux, and E. P. O'Reilly, J. Appl. Phys. 84, 297 (1999).

${ }^{14}$ F. Szmulowicz, Phys. Rev. B 51, 1613 (1995).

${ }^{15}$ S. L. Chuang, Physics of Optoelectronic Devices (Wiley, New York, 1995), page. 387.

${ }^{16}$ D. Ahn, Prog. Quantum Electron. 21, 249 (1997), see Fig. 3

${ }^{17}$ E. P. O'Reilly, Semicond. Sci. Technol. 4, 121 (1989). 